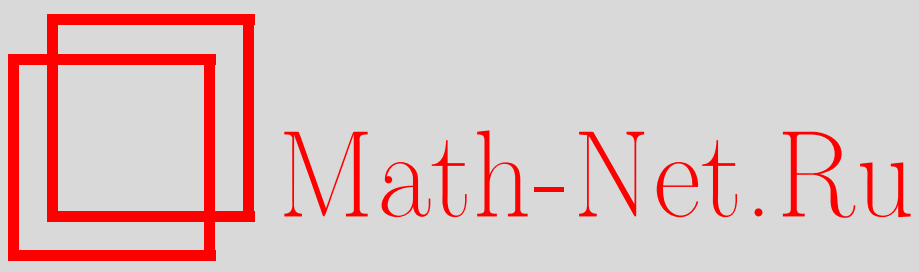

Р. С. Исмагилов, Ш. Ш. Султанов, Нормализованное семейство представлений группы движений евклидова пространства и обратная задача теории представлений этой группы, Матем. сб., 2004, том 195, номер 12, 47-56

DOI: https://doi.org/10.4213/sm864

Использование Общероссийского математического портала Math-Net.Ru подразумевает, что вы прочитали и согласны с пользовательским соглашением

http: //www.mathnet.ru/rus/agreement

Параметры загрузки:

IP: 54.80 .73 .141

26 апреля 2023 г., 12:48:05 


\title{
Нормализованное семейство представлений группы движений евклидова пространства и обратная задача теории представлений этой группы
}

\begin{abstract}
Хорошо известно голоморфное семейство $\mathscr{T}^{\lambda}$ представлений группы изометрий пространства $\mathbb{R}$ такое, что $\mathscr{T}^{-\lambda} \sim \mathscr{T}^{\lambda}$ при $\lambda \neq 0$. В статье указано голоморфное семейство $V_{R}^{\lambda},|\lambda|<R$, такое, что $V_{R}^{\lambda} \sim \mathscr{T}^{\lambda}$ и $V_{R}^{-\lambda}=V_{R}^{\lambda}$ при $\lambda \neq 0$. Это используется для построения представлений (вообще говоря, приводимых) достаточно общего вида.

Библиографиял: 10 названий.
\end{abstract}

\section{Введение. Постановка задачи и краткое описание результатов}

Элементы групшы $G=\operatorname{Iso}\left(\mathbb{R}^{n}\right)$, указанной в названии статьи, записываются в виде $g=(u, a)$, где $u \in \mathrm{SO}(n), a \in \mathbb{R}^{n}$, а групповая операция имеет вид $\left(u_{1}, a_{1}\right)\left(u_{2}, a_{2}\right)=\left(u_{1} u_{2}, a_{1}+u_{1} a_{2}\right)$. Для любого комплексного числа $\lambda$ определено линейное представление этой группы, действующее в гильбертовом пространстве $L_{2}\left(S^{n-1}\right)$ (здесь $S^{n-1}$ означает сферу $\left.\{x:|x|=1\}\right)$ по формуле

$$
\mathscr{T}^{\lambda}(g) f(x)=\exp (\lambda(a, x)) f\left(u^{-1} x\right), \quad x \in S^{n-1}, \quad f \in L_{2}\left(S^{n-1}\right) .
$$

Оно неприводимо при $\lambda \neq 0$ и унитарно при $\operatorname{Re} \lambda=0$. Кроме того, представления $\mathscr{T}^{\lambda}, \mathscr{T}^{-\lambda}$ эквивалентны (для краткости пишем $\mathscr{T}^{\lambda} \sim \mathscr{T}^{-\lambda}$ ); это означает, что $\mathscr{T}^{\lambda}(g) A=A \mathscr{T}^{-\lambda}(g)$ для некоторого линейного оператора $A$, осуществляющего линейньй гомеоморфиизмпространства $C^{\infty}\left(S^{n-1}\right)$ (относительно его естественной топологии).

Нам понадобится оператор Лапласа $\Delta$ на $G$; он имеет вид

$$
\sum_{k=1}^{n}\left(\frac{\partial}{\partial a_{k}}\right)^{2}, \quad g=(u, a) .
$$

Эти простые сведения изложены, например, в [1; гл. 11].

Первая цель этой статьи - построить новое семейство представлений группы $U^{\lambda}$ в том же пространстве $L_{2}\left(S^{n-1}\right)$, голоморфно зависящее от $\lambda \in \mathbb{C}$ и такое, что $U^{\lambda}=U^{-\lambda}$ для всех $\lambda \in \mathbb{C}$ (эквивалентность заменилась на равенство!) и $\mathscr{T}^{\lambda} \sim U^{\lambda}$ при $\lambda \neq 0$. Это и есть нормализованное представление. Строится также аналогичное семейство $V_{R}^{\lambda}$, определенное в полосе $|\operatorname{Re} \lambda|<R$ (здесь $R$-произвольное положительное число), обладающее некоторыми дополнительными достоинствами;

Работа первого автора поддержана Российским фондом фундаменталњных исследований (грант № 01-01-00490).

(C) Р.С. ИСмАГИЛОв, Ш.Ш. СУЛтАнов 2004 
оно более приспособлено для достижения второй (описываемой ниже) цели этой статьи.

Вторая наша цель - изучить произвольное представление $g \mapsto T(g), g \in G$, в гильбертовом пространстве $H$ такое, что

а) оно унитарно на подгруппе $\mathrm{SO}(n)$;

б) подпространство $H_{0}=\{h \in H, T(u) h=h, u \in \mathrm{SO}(n)\}$ нетривиально;

в) $H_{0}$ порождает все пространство $H$.

Выполнения условия а) можно добиться введением нового скалярного произведения в $H$; условие в) означает, что минимальное подпространство, инвариантное относительно $T$ и содержащее $H_{0}$, совпадает с $H$. Кое-где мы требуем лишь выполнения условий а), б); в этом случае можно добиться выполнения условия в), заменив $H$ на наименњшее подпространство, содержашее $H_{0}$ и инвариантное относительно представления.

Всюду далее используются введенные сейчас обозначения.

Представления с описанными свойствами назовем сферическими (хотя это название применяют обычно к неприводимым представлениям).

Мы попытаемся описать “произвольное” (или, хотя бы, достаточно общее) представление этого класса. Чтобы уточнить постановку задачи, рассмотрим для любого представления $T$ со свойствами а) и б) образ оператора Лапласа $\Delta$ на группе $G$ при представлении $T$ (точнее, при соответствующем представлении универсальной обертьвающей алгебры, полученной из алгебры Ли нашей групшы). Ограничим его на $H_{0}$ и обозначим через $T_{0}(\Delta)$ замыкание этого ограничения. Итак, представлению $T$ мы поставили в соответствие линейный оператор $T_{0}(\Delta)$ в $H_{0}$ с плотной областью определения. Именно этот оператор мы возьмем в качестве "параметра", задающего сферическое представление. Свойства спектра и резольвенты оператора $T_{0}(\Delta)$ изучаются в $\S 2$; этим решается “прямая задача" теории представлений рассматриваемого класса. Резонно считать, что мы умеем строить “произвольное” (или, во всяком случае, достаточно общее) сферическое представление, если мы научились решать нижеследуюшую “обратную задачу”.

Пусть даны некоторое гильбертово пространство $H_{0}$ и действующий в нем линейный оператор $A$ с плотной областью определения. Требуется построить сферическое представление $T$ в более широком гильбертовом пространстве $H$ такое, что

$$
H_{0}=\{h \in H, T(u) h=h, u \in \mathrm{SO}(n)\}, \quad T_{0}(\Delta)=A .
$$

Разумеется, это выполнимо лишш при некоторых ограничениях на $A$, и в $\S 2$ указываются условия на $A$, необходимые для решения этой задачи. В $\S 4$ мы опишем достаточные условия (они не совпадут с необходимыми, но по духу будут близки к ним) и укажем явную конструкцию представления $T$. Именно для этой цели используется второе из упомянутых нормализованных семейств.

Исследования связи представлений группы Ли с соответствующими представлениями алгебры Ли имеют большую историю. Для простейшего случая аддитивной группы $\mathbb{R}$ вопрос сводится к описанию производящих операторов однопараметрических операторных групп. Эта задача была решена в работе И. М. Гельфанда [2]. В дальнейшем этот результат был перенесен на однопараметрические полугрупшы описание этих результатов и соответствующие ссылки содержатся 
в [3; гл. 7]. Для произвольной (односвязной) группы Ли указанный вопрос рассматривался в работе С. Г. Крейна, А. М. Шихватова [4]; в этой работе выяснялись условия на представление алгебры Ли (в банаховом пространстве), при выполнении которых представление можно “поднять" на группу.

В предлагаемой работе постановка задачи о связи между представлениями групшы Ли и ее алгебры претерпела сушественное изменение. Мы рассматриваем не представление алгебры Ли, а лиш образ лапласиана $\Delta$ (точнее, ограничение $A=T_{0}(\Delta)$ этого образа на подпространство $\left.H_{0}\right)$; мы стремимся "восстановить" представление группы по оператору $A$. Можно сказать, что вместо традиционных уравнений первого порядка на группе здесь рассматриваются уравнения второго порядка.

Впервые нормализованное семейство представлений было построено для группы $\mathrm{SL}(2, \mathbb{R})$ в работе Р.А. Кунзе и Е.М. Штейна [5]. Это было сделано для семейства представлений основной серии $T_{s}$ (мы придерживаемся обозначений из [6; гл. 7]), где параметр $s$ пробегает полосу $|\operatorname{Re} s|<1$. Нормализованное семейство Кунзе-Штейна обладает рядом дополнительных весьма замечательных свойств. Если взять произвольную полосу вида $|\operatorname{Re} s|<l, l>1$, то она содержит особые точки $s=1, s=-1$, что делает невозможной конструкцию этих авторов. Тем не менее, для произвольной полосы нормализация (отличная от той, которая была построена в [5]) возможна и в этом случае. Это было сделано в [7]. В статье [7] была также поставлена (и решена при помощи нормализации в произвольной полосе) "обратная задача" теории представлений; ее формулировка близка к той, какую мы описали выше для группы $G=\operatorname{Iso}\left(\mathbb{R}^{n}\right)$.

Заметим, что для групшы $G=\operatorname{Iso}\left(\mathbb{R}^{n}\right)$ (в отличие от $\operatorname{SL}(2, \mathbb{R})$ ) задача нормализации представлений решается крайне просто. Это и побудило авторов рассмотреть этот пример, не связанный с техническими трудностями.

Работа завершается приложением, в котором на элементарном примере иллюстрируется задача нормализации семейства линейных операторов.

\section{§1. Нормализованные семейства представлений группы $G$}

Возьмем на комплексной $\lambda$-плоскости открытую связную область $D$, содержащую точку $\lambda=0$ и симметричную относительно этой точки; таким образом, $-\lambda \in D$ при $\lambda \in D$. Возьмем, далее, функцию $\lambda \mapsto \omega(\lambda), \lambda \in D$, со следуюшими свойствами: она голоморфна в $D, \omega(-\lambda)=-\omega(\lambda)$ при $\lambda \in D, \omega^{\prime}(0) \neq 0$ и $\omega(\lambda) \neq 0$ при $\lambda \neq 0, \lambda \in D$. Обратимся к представлению (1) и для каждого $\lambda \in D$, $\lambda \neq 0$, определим линейный оператор $M(\lambda)$ в пространстве $L_{2}\left(S^{n-1}\right)$ формулами $M(\lambda) f=f$, если функция $f$ четная, $M(\lambda) f=\omega(\lambda) f$, если функция $f$ нечетная. Рассмотрим представление $g \mapsto V^{\lambda}(g)=M(\lambda) \mathscr{T}^{\lambda} M^{-1}(\lambda), g \in G$. Легкий подсчет показывает, что оно действует по формуле

$$
\begin{aligned}
V^{\lambda}(g) f(x)= & \left(\operatorname{ch}(\lambda(a, x))+\frac{1}{2}\left(\omega(\lambda)+\frac{1}{\omega(\lambda)}\right) \operatorname{sh}(\lambda(a, x))\right) f\left(u^{-1} x\right) \\
& +\frac{1}{2}\left(\omega(\lambda)-\frac{1}{\omega(\lambda)}\right) \operatorname{sh}(\lambda(a, x)) f\left(-u^{-1} x\right) \\
& f \in L_{2}\left(S^{n-1}\right), \quad g=(u, a) \in G .
\end{aligned}
$$


Мы видим, что функция $\lambda \mapsto V^{\lambda}(g)$ голоморфна всюду в области $D$ (а не только при $\lambda \neq 0$, хотя сплетающий оператор $M^{-1}(\lambda)$ имеет полюс при $\left.\lambda=0\right)$. Выполняется равенство $V^{\lambda}(g)=V^{-\lambda}(g), \lambda \in D$. Нормализованное семейство построено.

Описанная конструкция задается произвольной областью $D$ и функцией $\omega(\lambda)$, $\lambda \in D$, удовлетворяющими указанным условиям; можно положить, например, $D=\mathbb{C}, \omega(\lambda)=\lambda$, что приводит к нормализованному семейству $U^{\lambda}$, упомянутому во введении. Однако для решения "обратной задачи” (о ней пойдет речь ниже) нам понадобится нормализация весьма частного вида. Чтобы построить ее, зафиксируем число $R>0$ и в качестве указанной выше области $D$ возьмем полосу $D_{R}=\{\lambda:|\operatorname{Re} \lambda|<R\}$, а в качестве функции $\omega: D \rightarrow \mathbb{C}$ возьмем функцию

$$
\lambda \mapsto \omega_{R}(\lambda)=\lambda\left(R^{2}-\lambda^{2}\right)^{-1 / 2}, \quad|\operatorname{Re} \lambda|<R,
$$

где берется ветвь квадратного корня такая, что $\sqrt{1}=1$. Нормализованное семейство, построенное (по описанному выше правилу) по области $D_{R}$ и функции $\omega_{R}$, обозначим через $V_{R}^{\lambda}$.

$\Phi$ ункция $\lambda \mapsto V_{R}^{\lambda}(g)$ ограничена (по норме) в любой меньшей полосе $|\operatorname{Re} \lambda|<c$, $c<R$, для любого $g \in G$. (Этим же свойством обладает и исходное семейство $\mathscr{T}^{\lambda}$.)

Для дальнейшего мы несколько изменим параметризацию построенного семейства. С этой целью для любого $c>0$ обозначим через $\Gamma_{c}$ область на $z$-плоскости, полученную как образ полосы $|\operatorname{Re} \lambda|<c$ при отображении $z=\lambda^{2}$. Ясно, что

$$
\Gamma_{c}=\left\{z: z=x+y i, x<c^{2}-\frac{y^{2}}{4 c^{2}}\right\} \text {. }
$$

Вернемся к семейству $V_{R}^{\lambda}$. Так как функция $\lambda \mapsto V_{R}^{\lambda}(g)$ четна, то определено голоморфное (по $z$ ) семейство представлений $W_{R}^{z}=V_{R}^{\sqrt{z}}, z \in \Gamma_{R}$. (Выбор значения $\sqrt{z}$ безразличен.) Его можно записать в виде

$$
\begin{aligned}
& W_{R}^{z}(g) f(x)=\left(\operatorname{ch}(\sqrt{z}(a, x))+\frac{1}{2}\left(\omega_{R}(\sqrt{z})+\frac{1}{\omega_{R}(\sqrt{z})}\right) \operatorname{sh}(\sqrt{z}(a, x))\right) f\left(u^{-1} x\right) \\
&+\frac{1}{2}\left(\omega_{R}(\sqrt{z})-\frac{1}{\omega_{R}(\sqrt{z})}\right) \operatorname{sh}(\sqrt{z}(a, x)) f\left(-u^{-1} x\right) \\
& f \in L_{2}\left(S^{n-1}\right), \quad g=(u, a) \in G
\end{aligned}
$$

Предыдушее название "нормализованное представление" мы будем применять и к этому семейству. Именно оно будет использовано для решения “обратной задачи".

\section{§ 2. Оператор $T_{0}(\Delta)$, его резольвента и спектр}

Мы сохраняем обозначения для элементов группы и оператора Лапласа на $G$, введенные в начале работы.

Рассмотрим произвольное сфферическое представление $g \mapsto T(g), g \in G$, в гильбертовом пространстве $H$; мы также сохраняем обозначения, введенные при описании класса сферических представлений. Пусть $P_{0}$ - ортогональный проектор на $H_{0}$. Ясно, что оператор $P_{0} T((e, a)) P_{0}$ (здесь $e-$ единица группы $\mathrm{SO}(n)$ и $\left.a \in \mathbb{R}^{n}\right)$ 
зависит только от величины $r=|a|$; обозначим его через $F(r)$. Легко видеть, что $T_{0}(\Delta)=\left.\Delta F(r)\right|_{r=0}, r=|a|, g=(r, a)$.

Без труда проверяется, что для представлений $(1),(3)$ подпространства $H_{0}$ одномерны и

$$
\mathscr{T}_{0}^{\lambda}(\Delta)=\lambda^{2}, \quad\left(W_{R}^{z}\right)_{0}(\Delta)=z
$$

здесь операторы в одномерном пространстве отождествлены с числами.

В следующей теореме, посвященной свойствам оператора $T_{0}(\Delta)$, используется функция Макдональда $K_{\nu}(z)$; ее определение и свойства изложены в [8; гл. 5]. Используется также область $\Gamma_{c}$, заданная равенством (2).

TЕОРема 1. Cnектр оператора $T_{0}(\Delta)$ содержится в области вида $\Gamma_{c}(\partial л я$ некоторого $c>0)$. Для $z \notin \Gamma_{c}$ резольвента $R(z)=\left(T_{0}(\Delta)-z I\right)^{-1}$ выражается формулой

$$
R(z)=\frac{z^{(n-2) / 4}}{2^{(n-2) / 2} \Gamma\left(\frac{n}{2}\right)} \int_{0}^{\infty} F(r) K_{(n-2) / 2}(r \sqrt{z}) r^{n / 2} d r
$$

(используются голоморфные ветви корней из $z \notin \Gamma_{c}$, принимающие значение 1 при $z=1)$.

Справедливо неравенство

$$
\|R(z)\| \leqslant l|z|^{(n-3) / 4}|\operatorname{Re} \sqrt{z}|^{-(n+1) / 2}, \quad z \notin \Gamma_{c}
$$

с некоторой постоянной $l>0$.

ДоказАтЕЛЬСтво. Асимптотическое поведение функции $K_{\nu}(z)$ при $z \rightarrow \infty$ и при $z \rightarrow 0$ описьвается следующими соотношениями.

Если $\nu>0$, то

$$
\begin{array}{ll}
K_{\nu}(z) \sim\left(\frac{\pi}{2 z}\right)^{1 / 2} \exp (-z), & z \rightarrow \infty, \quad|\arg z|<\pi, \\
K_{\nu}(z) \sim 2^{\nu-1} \Gamma(\nu) z^{-\nu}, & z \rightarrow 0, \quad|\arg z|<\pi .
\end{array}
$$

Кроме того,

$$
K_{0}(z) \sim \ln \left(\frac{2}{z}\right), \quad z \rightarrow 0, \quad|\arg z|<\pi .
$$

Функция $F(r), r \geqslant 0$, удовлетворяет неравенству $\|F(r)\| \leqslant M \exp (c r)$ для некоторых $M, c \geqslant 0$ (следствие стандартной оценки нормы однопараметрической группы операторов). Отсюда получаем (оценивая норму подинтегральной функции), что интеграл сходится при $\operatorname{Re} \lambda>c$, т.е. при $z \notin \Gamma_{c}$ (напомним, что $z=\lambda^{2}$ ). Отсюда вытекает также неравенство (6).

Формула (5) для резольвенты вытекает из того, что функция

$$
E(r)=\frac{\left(\frac{\nu}{2}\right)^{(n-2) / 2}}{\Gamma\left(\frac{n}{2}\right)} r^{-(n-2) / 2} K_{(n-2) / 2}(\nu r), \quad r=|x|, \quad x \in \mathbb{R}^{n}
$$


является фундаментальным решением оператора Гельмгольца $\Delta-\nu^{2} I$ (см. [9; гл. 3]). Более точно, это - главное фундаментальное решение; оно выделяется тем, что стремится к нулю при $r \rightarrow \infty$. Теорема 1 доказана.

Итак, мы получили некоторые свойства резольвенты и спектра оператора $T_{0}(\Delta)$. В $\S 4$ мы увидим, что близкий по духу набор требований, наложенных на оператор $A$ в гильбертовом пространстве $H_{0}$, достаточен для того, чтобы этот оператор мог служить в качестве $T_{0}(\Delta)$ для некоторого представления $g \mapsto T(g), g \in G$. Таким образом, будет решена (при некоторых ограничениях) "обратная задача" теории представлений для группы $G$. Но предварительно (в $\S 3$ ) мы обсудим способы построения "достаточно общих" представлений того класса, который был описан в начале $\S 2$. В частности, мы объясним роль нормализованного семейства для решения нашей задачи.

\section{§3. Конструкция некоторых представлений группы, необходимость нормализации}

Возьмем сначала исходное (ненормализованное) семейство (1). Как построить, используя его, представление $g \mapsto T(g), g \in G$, достаточно общего вида? Вполне естественным представляется следующий способ. Взять гильбертово пространство $H=L_{2}\left(S^{n-1}, H_{0}\right)$ вектор-функций со значениями в некотором гильбертовом пространстве $H_{0}$, взять затем оператор $B$ (скажем, ограниченньй) в $H_{0}$ и задать в $H$ представление формулой

$$
T(g) f(x)=\exp ((x, a) B) f\left(u^{-1} x\right), \quad x \in S^{n-1} .
$$

Естественно обозначить это представление через $g \mapsto \mathscr{T}^{B}(g), g \in G$. Мы попросту "подставили" оператор $B$ вместо переменной $\lambda$. Ясно, что подпространство $\left\{h: h \in H, \mathscr{T}^{B}(u) h=h, u \in \mathrm{SO}(n)\right\}$ отождествляется с $H_{0}$, а оператор $\mathscr{T}_{0}^{B}(\Delta)$ сводится к $B^{2}$; последнее доказывается посредством тех же простых выкладок, которые приводят к первому из равенств (4). Таким образом, в качестве $\mathscr{T}_{0}^{B}(\Delta)$ выступает оператор, обладающий квадратным корнем. Известно, однако, что существуют ограниченные операторы, не имеющие квадратных корней (отметим легко доказываемое свойство такого оператора: его резольвентное множество не может содержать непрерьвной кривой, соединяющей нуль с бесконечностью). Итак, семейство (1) не позволило решить "обратную задачу" (даже для ограниченных операторов). Поэтому резонно "подставить" оператор $B$ вместо переменной не в исходное семейство (1), а в нормализованное семейство (3). При этом образом оператора Лапласа (в соответствующем подпространстве $H_{0}$ ) будет в точности оператор $B$ (а не его квадрат!); это доказывается посредством выкладок, даюших второе из равенств (4). О том, каким образом осушествляется указанная "подстановка", будет сказано в следуюшем параграфе.

\section{§4. Решение "обратной задачи"}

Пусть $H_{0}$ - гильбертово пространство и $A$ - замкнутый линейный оператор с плотной областью определения. Пусть спектр оператора $A$ содержится в области вида $\Gamma_{c}$ (заданной формулой $(2)$ для некоторого $c>0$ ). (Необходимость этого ограничения доказана в теореме 1.) Мы должны указать достаточные условия, при которых $T_{0}(\Delta)=A$ для некоторого представления $T$ группы $G$. 
Как уже разъяснялось, оператор $A$ может не иметь квадратного корня (если даже он ограничен). Однако для любого $R>c$ спектр оператора $A-R^{2} I$ заключен в области $\Gamma_{c}-R^{2}$, а потому содержится внутри угла с вершиной $z=0$ и раствора меньше $\pi$, расположенного в левой полуплоскости. Но для таких операторов сушествование квадратного корня из $A-R^{2} I$ уже не является насильственным требованием; скажем, любой ограниченный оператор с таким спектром имеет квадратный корень. Теория дробных степеней операторов (изложенная, например, в книге М. А. Шубина [10; гл. 2]) позволяет строить нужный корень также для неограниченных операторов при незначительных ограничениях. (Отметим, что в этой книге рассматриваются операторы, спектр которых лежит внутри угла, расположенного в правой полуплоскости.)

Итак, мы налагаем на оператор следуюшие два ограничения.

а) Для некоторого $R>c$ определен оператор $T=\sqrt{A-R^{2} I}$ в смысле теории дробных степеней операторов. Для определенности здесь мы используем ту голоморфную в области $\mathbb{C} \backslash[0, \infty)$ ветвь квадратного корня $\sqrt{z}$, для которой $\sqrt{-1}=i$; эта ветвь будет использоваться и в дальнейшем без дополнительных разъяснений. Указанная функция отображает $\Gamma_{c}-R^{2}$ на часть полуполосы $\{z:|\operatorname{Re} z|<c, \operatorname{Im} z>0\}$. Таким образом, спектр оператора $T$ заключен в этой полуполосе.

б) Оператор $T$ служит производящим оператором однопараметрической группы (запишем ее, как обычно, в виде $t \mapsto \exp (t T)$ ), и выполняется неравенство

$$
\|\exp (t T)\| \leqslant M e^{c|t|}, \quad t \in \mathbb{R}
$$

В силу указанного расположения спектра оператора $T=\sqrt{A-R^{2} I}$ условие б) достаточно естественно. Используемые ниже сведения об однопараметрических группах и их производящих операторах содержатся в [9; гл. 7].

Докажем, что условия а) и б) достаточны для решения "обратной задачи".

Обозначим через $\mathscr{F}_{R}$ алгебру (относительно умножения) функций $f(z), z \in \Gamma_{R}$, удовлетворяющих следующим условиям:

i) $f$ голоморфна в области $\Gamma_{R}$;

ii) для любого целого $n>0$ справедливо асимптотическое разложение вида

$$
\begin{gathered}
f(z)=\sum_{k} a_{k} \exp \left(b_{k} \sqrt{z}\right) p_{k}\left(\frac{1}{\sqrt{z}}\right)+o\left(z^{-n}\right), \\
z \in \Gamma_{R}, \quad \operatorname{Re} z \rightarrow-\infty, \quad b_{k} \in \mathbb{R}
\end{gathered}
$$

сумма конечна, $p_{k}$ - многочлены.

Сейчас мы построим "операционное исчисление" на алгебре $\mathscr{F}_{R}$, т.е. естественньй гомоморфизм из $\mathscr{F}_{R}$ в алгебру $B\left(H_{0}\right)$ ограниченных операторов в $H_{0}$.

Для этого заметим, что из (8) вытекает также асимптотическое представление

$$
\begin{gathered}
f(z)=\sum_{k} \alpha_{k} \exp \left(\beta_{k} \sqrt{z-R^{2}}\right) q_{k}\left(\frac{1}{\sqrt{z-R^{2}}}\right)+o\left(z^{-n}\right), \\
z \in \Gamma_{R}, \quad \operatorname{Re} z \rightarrow-\infty, \quad \beta_{k} \in \mathbb{R},
\end{gathered}
$$


сумма конечна, $q_{k}-$ многочлены. Это следует из того, что функция $\sqrt{z}$ разлагается в асимптотический ряд по степеням $\left(z-R^{2}\right)^{-j+1 / 2}, j=0,1, \ldots$, при $|z| \rightarrow \infty$, $z \in \Gamma_{R}$, и из того, что функция $\exp (b \sqrt{z}), b \in \mathbb{R}$, ограничена при $z \in \Gamma_{R}, \operatorname{Re} z<0$.

Используя соотношение (9), легко построить ограниченньй оператор $f(A)$ для любой функции $f \in \mathscr{F}_{R}$. Во-первых, операторы $\exp \left(\beta \sqrt{A-R^{2} I}\right), \beta \in \mathbb{R}$, $\left(A-R^{2} I\right)^{-1 / 2}$ определены (как ограниченные операторы) естественным образом (согласно условиям i), ii)). Далее, для любой функции $f \in \mathscr{F}_{R}$ такой, что $f(z)=o\left(z^{-2}\right), \operatorname{Re} z \rightarrow-\infty$, оператор $f(A)$ определяется стандартной формулой

$$
f(A)=\frac{1}{2 \pi i} \int_{L_{l}} f(z)(A-z I)^{-1} d z,
$$

где $L_{l}$ - образ прямой $\operatorname{Re} \lambda=l$ при отображении $z=\lambda^{2}$ и $c<l<R$. (Кривая $L_{l}$ ориентирована так, что при движении по ней область $\Gamma_{l}$ остается слева.)

Чтобы доказать сходимость (по норме) этого интеграла, докажем, что величина $\left\|(A-z I)^{-1}\right\|, z \in L_{l}$, ограничена. Предварительно напомним, что из неравенства (7) вытекают неравенства

$$
\left\|(T-\lambda I)^{-1}\right\| \leqslant M|| \operatorname{Re} \lambda|-c|^{-1}, \quad|\operatorname{Re} \lambda|>c .
$$

Несложный вывод содержится, например, в [3; гл. 7].

Пусть $z \in L_{l}, z=\lambda^{2}$, Re $\lambda=l$. Тогда $A-z I=T^{2}+R^{2} I-\lambda^{2} I=\left(T-\lambda_{1} I\right) \times$ $\left(T+\lambda_{1} I\right)$, где $\lambda_{1}^{2}=\lambda^{2}-R^{2}, \operatorname{Re} \lambda_{1}>0$. Согласно неравенству (11)

$$
\|(A-z I)^{-1}|| \leqslant M^{2}|| \operatorname{Re} \lambda_{1}|-c|^{-2} \text {. }
$$

Если $\operatorname{Re} \lambda=l$ и величина $|\lambda|$ достаточно велика, то величина $\operatorname{Re} \lambda_{1}$ сколь угодно близка к $\operatorname{Re} \lambda=l$. Отсюда $\left\|(A-z I)^{-1}\right\| \leqslant M_{1}(l-c)^{-2}$, чем доказана ограниченность величины $(A-z I)^{-1}, z \in L_{l}$.

Теперь сходимость интеграла (10) очевидна.

Итак, построены операторы $f(A), A \in \mathscr{F}_{R}$. Несложную проверку того, что в итоге получился гомоморфизм $\mathscr{F}_{R} \rightarrow B\left(H_{0}\right)$, опускаем.

Для нас существенно следующее свойство построенного “операционного исчисления". Как известно, определено плотное линейное подмножество $\mathscr{L} \subset H_{0}$, состоящее из аналитических векторов группы $t \mapsto \exp (t T)$; оно назьвается пространством Гординга. Операторы $T, T^{2}$ являются замьканиями своих ограничений на $\mathscr{L}$. Операторы $f(A), A \in \mathscr{F}_{R}$, отображают $\mathscr{L}$ на себя. Операторнозначные функции $s \mapsto f(s A)$, примененные к векторам из $\mathscr{L}$, можно неограниченно дифференцировать по $t$. Эти факты изложены в [3; гл. 7], а также в [4].

Теперь легко "подставить" оператор $A$ вместо $z$ в нормализованное семейство (3). Действительно, функции $\operatorname{ch}(\sqrt{z}), \omega_{R}(\sqrt{z}) \operatorname{sh}(\sqrt{z}),\left(\omega_{R}(\sqrt{z})\right)^{-1} \operatorname{sh}(\sqrt{z})$ очевидным образом принадлежат алгебре $\mathscr{F}_{R}$; следовательно, мы можем подставить в них $A$ вместо $z$. Рассмотрим гильбертово пространство $H=L_{2}\left(S^{n-1}, H_{0}\right)$ и зададим в нем операторы

$$
\begin{aligned}
& W_{R}^{A}(g) f(x)=\left(\operatorname{ch}(\sqrt{A}(a, x))+\frac{1}{2}\left(\omega_{R}(\sqrt{A})+\frac{1}{\omega_{R}(\sqrt{A})}\right) \operatorname{sh}(\sqrt{A}(a, x))\right) f\left(u^{-1} x\right) \\
&+\frac{1}{2}\left(\omega_{R}(\sqrt{A})-\frac{1}{\omega_{R}(\sqrt{A})}\right) \operatorname{sh}(\sqrt{A}(a, x)) f\left(-u^{-1} x\right), \\
& f \in L_{2}\left(S^{n-1}\right), \quad g=(u, a) \in G .
\end{aligned}
$$


Они образуют представление группы $G$.

(Разумеется, мы допустили некоторую нестрогость, используя обозначение $\sqrt{A}$ в этих формулах, так как $\sqrt{A}$ может не сушествовать; выше мы уже объяснили точный смысл приведенных формул.)

Подпространство $\left\{h: h \in H, W_{R}^{A}(u) h=h, u \in \mathrm{SO}(n)\right\}$ состоит из постоянных вектор-функций и, таким образом, отождествляется с $H_{0}$. Обозначим через $\widetilde{W}_{R}^{A}$ ограничение представления $W_{R}^{A}$ на наименьшее инвариантное подпространство, содержашее $H_{0}$.

Мы пришли к следуюшей теореме, решаюшей “обратную задачу” для заданного оператора $A$.

Теорема 2. Для сферического представления $\widetilde{W}_{R}^{A}$ справедливо равенство $\left(\widetilde{W}_{R}^{A}\right)_{0}(\Delta)=A$.

Для доказательства следует применить оператор Лапласа на группе $G$ к вектор-функции $g \mapsto W_{R}^{A}(g) h, h \in \mathscr{L}$, в точке $g=e$, используя предыдущее замечание о бесконечной дифференцируемости этой вектор-функции; это сводится к несложньмм выкладкам.

\section{Приложение}

Здесь приводится весьма простой пример, иллюстрируюший идею нормализации семейства операторов (не представлений).

Пусть группа Г действует голоморфными преобразованиями $x \mapsto x \gamma$ на связном комплексном многообразии $X$. Пусть $f(x), x \in X$, - голоморфная функция со значениями в пространстве комплексных матриц $M_{n}$. Предположим, что на некотором открытом подмножестве выполнено условие $f(x) \sim f(x \gamma), \gamma \in \Gamma$, и матрица $f(x)$ имеет только простые собственные значения. Тогда существует такая голоморфиная функция $x \mapsto f_{1}(x), x \in X$, со значениями в $M_{n}$, что $f(x) \sim f_{1}(x)$ на открытом плотном множестве и $f_{1}(x)=f_{1}(x \gamma)$ для всех $\gamma \in \Gamma, x \in X$.

Для доказательства рассмотрим функцию $d(x, \lambda)=\operatorname{det}(f(x)-\lambda I)=(-1)^{n} \lambda^{n}+$ $d_{n-1}(x)+\cdots+d_{0}(x)$. Матрица

$$
\left(\begin{array}{ccccc}
0 & 0 & \ldots & 0 & (-1)^{n-1} d_{0}(x) \\
1 & 0 & \ldots & 0 & (-1)^{n-1} d_{1}(x) \\
\vdots & \vdots & \ddots & \vdots & \vdots \\
0 & 0 & \ldots & 1 & (-1)^{n-1} d_{n-1}(x)
\end{array}\right)
$$

искомая.

\section{Список литературы}

1. Виленкин H. $Я$. Специалњные функции и теория представлений групп. М.: Наука, 1991.

2. Гельфанд И. М. Об однопараметрических группах операторов в нормированном пространстве // Докл. АН СССР. 1939. Т. 25. №9. С. 711-716.

3. Данфорд Н., Швари Джс. Т. Линейные операторы. Общая теория. М.: ИЛ, 1962.

4. Крейн С. Г., Шихватов А. М. Линейнше дифференциальные уравнения на группе Ли // Функц. анализ и его прилож. 1970. Т. 4. №1. С. 52-61.

5. Kunze R.A., Stein E.M. Uniformly bounded representations and harmonic analysis on the $2 \times 2$ unimodular group // Amer. J. Math. 1960. V. 82. № 1. P. 1-62. 
6. Гельфанд И. М., Граев М. И. Интегральная геометрия и связанные с ней вопросы теории представлений. М.: Физматгиз, 1962.

7. Исмагилов Р. С. О линейных представлениях группы $\operatorname{SL}(2, \mathbb{R})$ // Матем. сб. 1967. T. 74 (116). № 4 . C. 496-515.

8. Лебедев Н. Н. Специальные функции и их приложения. М.: Физматгиз, 1963.

9. Михлин С. Г. Линейные уравнения математической физики. М.: Наука, 1964.

10. Шубин М. А. Псевдодифференциальные операторы и спектральная теория. М.: Наука, 1978.

Московский государственнњй

технический университет им. Н.Э. Баумана;

Поступила в редакцию

Нижневартовский педагогический институт 